\title{
Evaluation of the Asymptotic Field at Interfaces of Ductile Materials
}

\section{ROSHDY S. BARSOUM}

US Army Materials Technology Laboratory, Watertown, Massachusetts 02172-0001, USA

\section{ABSTRACT}

This paper discusses the application of the Finite Element Iterative Method (FEIM) for the evaluation of the asymptotic fields at interfaces of dissimilar materials with power law hardening materials.

\section{KEYWORDS}

Asymptotic Field; Interfaces; Singularities; Finite clements IUTRODUCTION

Renewed interest in the problem of interfacial cracks [1-4] was realized due to recent advances in the development of materials such as ceramics, organic composites, adhesives, and micromechanics of failure in advanced materials.

The need for characterization of the asymptotic field at an interface is fundamental for the development of the test and design parameters. Little progress has been made in the characterization of the asymptotic field at an interface of elastic-plastic materials. For example, reference [5] gives an elastic-plastic analysis of a material bonded to a substrate. conclude that the asymptotic field is non-separable.

The author has recently applied the Finite Element Iterative Method (FEIM) to form rigid substrate $[10,11]$. The results show that a separable oscillatory solution similar to the elastic case [1,2], Can describe this field. The real part of the singularity is close to the part depends weakly on the hardening power. 
The FEIM was originally developed for the solution of

singularity problems in elastic media $[8,9]$. It is based on the fact that the asymptotic field can be expressed as a separable function $h(r) f(\theta)$. Where, for the stresses and strains, $h(r)$ is singular in $r$ and $f(\theta)$ is a regular

Using this fact, a circular fan finite element mesh is constructed, Figure 1, around the singularity. An iterative scheme is then carried out by imposing displacements on the outer boundary $\left\{u_{R_{0}}\right\}$ which are found from the resulting displacements $\left\{u_{R_{s}}\right\}$ at an inner radius $R_{s}$ in the previous iteration. The rigid body motion is subtracted from \{uss\} and the resulting displacements are scaled by a factor $\hat{R}_{\text {to }}$ keep, e.g.', the same

$$
[T]\left\{\tilde{\sim}_{R_{b}}^{n}\right\}=\left\{\sim_{R_{s}}^{n}\right\} \quad,\left\{\sim_{R_{b}}^{n+1}\right\}=\Lambda\left\{u_{R_{s}}^{n}-u_{0}^{n}\right\}
$$

\section{where [T] is the transfer matrix between the boundary}

displacements $\left\{u_{R_{b}}^{n}\right\}$ and the inner ring displacements $\left\{u_{R_{s}}^{n}\right\}$.

Depending on the form of $h(r)$, the scalling factor $\Lambda$ will ither reach a constant value or oscillate after many terations. After $n$ iterations, convergence is judged by the following condition:

$$
\left\{\sim_{R_{s}}^{n}\right\}=\alpha\left\{\sim_{R_{s}}^{j}\right\}+\beta\left\{\underset{\sim}{u_{R_{s}}^{k}}\right\} \quad \begin{aligned}
& j, k>n \\
& \alpha, \beta=\text { const. }
\end{aligned}
$$

This condition is derivable from the fact that the FEIM is based on the Rayleigh quotient and that after convergence, the vector $\left\{u_{R}^{n}\right\}$ can be expressed in terms of the dominant

eigenfunction and its conjugate [9] i.e.

$$
\left\{{\underset{\sim}{R_{s}}}_{n}^{n}\right\}=\alpha_{1} \underset{\sim}{x_{1}}+\bar{\alpha}_{1} \bar{x}_{1}
$$

where $\underset{\sim}{x_{1}},{\underset{\sim}{x}}_{1}$ are the first dominant eignefunction and its

conjugate, $\alpha_{1}$ and $\bar{\alpha}_{1}$ are conjugate constants. In the case of power law hardening materials, equation (1) is written in terms of the tangent stiffness matrix of the circular domain in Figure 1 and the incremental displacements, rather than

the elastic stiffness and the total displacements. Equation (1) therefore becomes;

$$
\begin{aligned}
& {\left[T\left(\sim_{\sim}^{p}\right)\right]\left\{\delta \sim_{R_{b}}^{n}\right\}=\left\{\delta \sim_{R_{s}}^{n}\right\}} \\
& \left\{\delta \underset{\sim}{\left.u_{R_{b}}^{n+1}\right\}=\Lambda}=\Lambda{\underset{\sim}{R_{s}}}^{n}-\delta \sim_{0}^{n}\right\}
\end{aligned}
$$

The displacements ( $u^{P}$ ) used in evaluating the tangent stiffnps substructuring of a stiffness matrix are same mesh, of Figure lb, fully plastic dis substructuring investigation of the asustructure number is approaches the $\mu$ and designated as $\mu$ and the proted is given by $R_{p}=\left(R_{i} / R_{b}\right)^{\mu}$ asymptotic field $R_{i}$ is the substructure radius and $R_{b}$,

is the outer radius.

EVALUATION OF THE ASYMPTOTIC FIELD FOR POWER LAW HARDENING EVALUATION OF THE AST ON THE INTERFACE OF A RIGID SUBSTRATE

(he FEIM gives the In references $[10,11]$, we have shown that the respotic field following form for the displacementaw hardening material and a rigid interface.

$$
\underset{\sim}{u}\left(R_{p}\right)=R_{e}\left\{\left(k_{1}^{p}+i k_{2}^{p}\right) r^{\left[a\left(R_{p}\right)+i \varepsilon\left(R_{p}\right)\right]} \bar{\sim}(\theta)\right\}
$$

here $\bar{u}(\theta)$ is a where larger than the HRR value of $(1 /(1+n))$, and changes as the process zone gets smaller. "n", here, is the hardening powe pr the stress in the Ramberg-Osgood stress-strain relationship. The imaginary part $\varepsilon$ is given in references $[10,11]$ and was shown to be weakly dependent on the hardening powe and the process zone size. Figure 2 gives " $a$ " and $\varepsilon$ as "a function of the process zone size vs. the hardening " $n$ ". Applying Eq. 2 to the displacement fields resulting from consecutive process zones $\mu_{1} \mu_{2}$,.... that $x^{\mu+1}=(\xi+i \eta) x^{\mu}, u=\operatorname{Re}\{\underset{\sim}{x}\}$

$$
x^{\mu}=\eta \sim_{\sim}^{\mu}+i\left(\xi \sim_{\sim}^{\mu}-k_{\mu+1} u^{\mu+1}\right)
$$

where $\mu^{\mu}=u\left(R_{p}\right)$ is given by Eq. 5 at the corresponding substructure $\mu$. $\xi$ and $\eta$ are weekly dependent on the hardening 
power $\mathrm{n}$ and $k_{\mu+1}$ is a scaling factor for the substructure. For $n=3, \xi=0.9556, \eta=0.2716$ and $n=5, \xi=0.9188$ and $\eta=0.2362$.

Thus the full asymptotic field can be described by equations 5 and 6. The use of Eq.(6), leads to an error in Eq.(2) less than 48 at the $\left(10^{-12}\right)$ process zone, which could be due to the large number of computations in the nonlinear substructuring analysis.

The relationships between the stresses and strains are obtained from analytical considerations of differentiating of the displacements and the value of the J-integral which is evaluated in the course of finding the tangent stiffness matrix. The J-integral in all calculations of rigid substrate was found to be constant. Following reference [6], if one could show numerically that the strain energy density around a circular contour to be given by

$$
\sigma_{i j} \epsilon_{i j} \longrightarrow \frac{f(\theta)}{r}
$$

then a full description of the asymptotic field can be obtained. Figures 3 and 4 give a plot of the strain energy density multiplied by the radial distance versus the radial distance. It is clear that the $\left(\sigma_{i j} \epsilon_{i j} \cdot r\right)$ is only a

function of $\theta$ once we get away from the crack tip. The reason for the oscillations near the crack tip is that the constant strain elements are not able to represent the oscillatory asymptotic field of equation ( 5 ).

From equations $(5,6$, and 7$)$, the Ramberg-Osgood stress-strain relationship and differentiating the displacements, we get

$$
\begin{aligned}
& \epsilon_{i j}\left(R_{p}\right)=R_{e}\left\{\left(k_{1}^{P}+i k_{2}^{p}\right)\left[a\left(R_{p}\right)-1+i \varepsilon\left(R_{p}\right)\right] \bar{\epsilon}_{i j}(\theta)\right\} \\
& \sigma_{i j}\left(R_{p}\right)=R_{e}\left\{\left(k_{1}{ }^{p}+i k_{2}^{p}\right)_{r}\left[-a\left(R_{p}\right)-i \varepsilon\left(R_{p}\right)\right] \overline{\sigma_{i j}}(\theta)\right\}
\end{aligned}
$$

where $\bar{\epsilon}_{i j}(\theta), \bar{\sigma}_{i j}(\theta)$ are complex functions of $\theta$, they are found by the approach given in reference [9] rather than differentiation.

It should be noted here that equations (5-8) are representative of the asymptotic field in a least square sense. The degree of approximation depends on how Eq. 2 is satisfied and the descritization errors encountered in the finite element idealization for calculating th
stiffness matrix and the transfer matrix [T].
CONCLUSIONS

The Finite Element Iterative Method (FEIM) was shown to be capable of dealing with nonlinear problems as well as linear problems. The asymptotic field for interfacial cracks with power law hardening materials was shown to be represented by a weak oscillatory function, similar to the elastic case of a crack at an interface. The real and imaginary parts however depend weakly on the size of the process zone. The full field can be written in a complex form of a recursive formula, once two consecutive process zones are evaluated.

The strain energy density was shown to be singular of power and behaves as $1 / r$ and the J-Integral to be constant, similar to the HRR-field. The FeIM leads to separable asymptotic fields in the least square sense.

\section{REFERENCES}

(1) Williams, M. L. (1959). The stresses around a fault or crack in dissimilar media. Bull. Seism.Soc. Am., 49, 199-204.

(2) Rice, J. R. and Sih, G. C. (1965). Plane problems of

cracks in dissimilar media. J. Appl. Mech., 32, 418-423. Comninou, M. (1977). The interface crack. J. Appl. Mech. ASME, 44, 631-636.

(4) IUTAM, Cracks in Bimaterial Interfaces, "Recent Advances

5) Shih, C. F. and Asaro, R. J. (March 1987).

Elastic-Plastic analysis of cracks on bimaterial

interfaces Part I- Small scale yielding. Brown University

(6) Rice, J. R. and Rosengren, G. F. (1968). Plane strain deformation near a crack tip in a power law hardening material. J. Mech. Phys. Solids, 16, 1-12.

(7) Hutchinson, J. W. (1969). Singular behavior at the end of a tensile crack in a hardening material. J. Mech. Phys. Solids, 16, 13-31.

(8) Barsoum, R. S. (1988) Theoretical basis of the finite element iterative method for the eigenvalue problem in stationary cracks. Int. J. Num. Meth. Eng.', 25, 531-539.

(9) Barsoum, R. S.(1988) Application of the finite element iterative method tissimilar media. Int. J. Num. Meth. Eng., 25, between
$541-554$

(10) Barsoum, R. S.(1988) On the separability of the asymptotic field at an interface crack. To appear. (Notes) Int. J. Fract..

(11) Barsoum, Int. J. Fract.. Submitted for Publication, singular behavior near an interface crack tip of power law hardening materials using the finite element iterative method. 


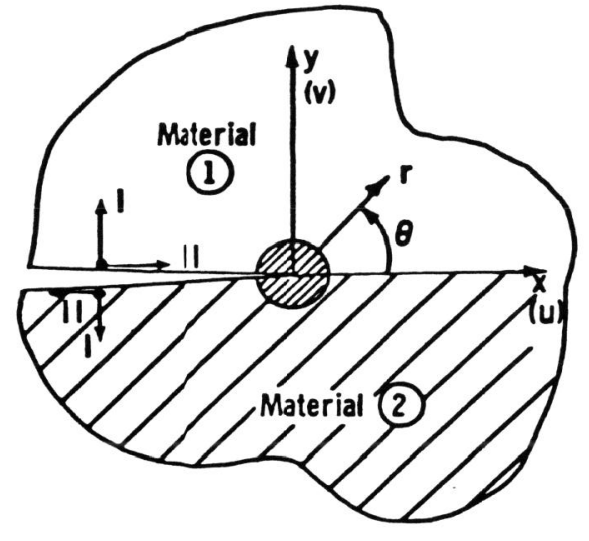

Fig. la. Interface Crack.

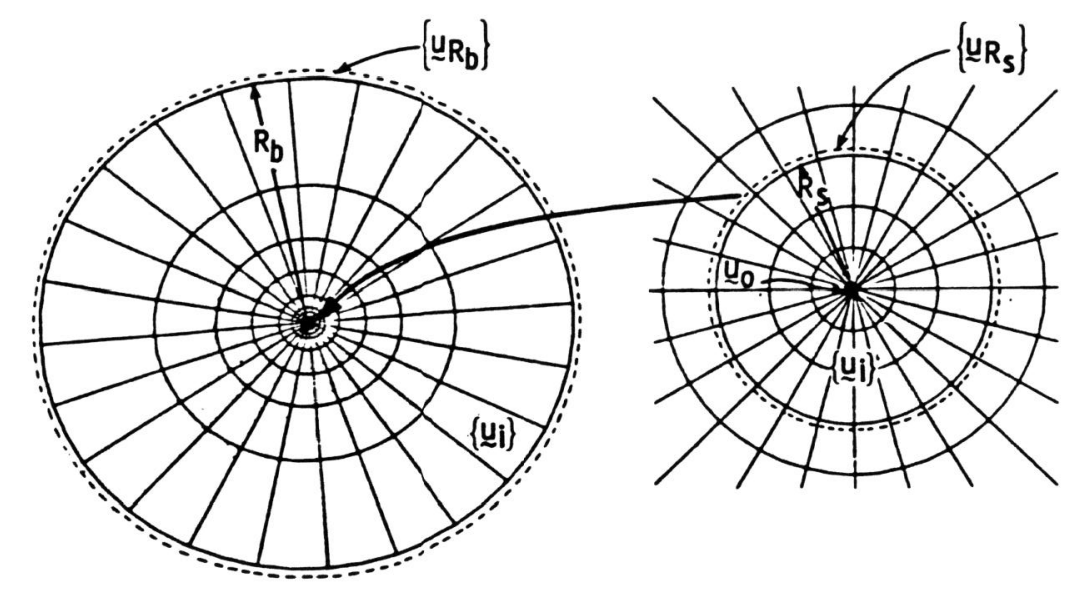

Fig. 1b. Finite Element Mesh for Iterative Method.
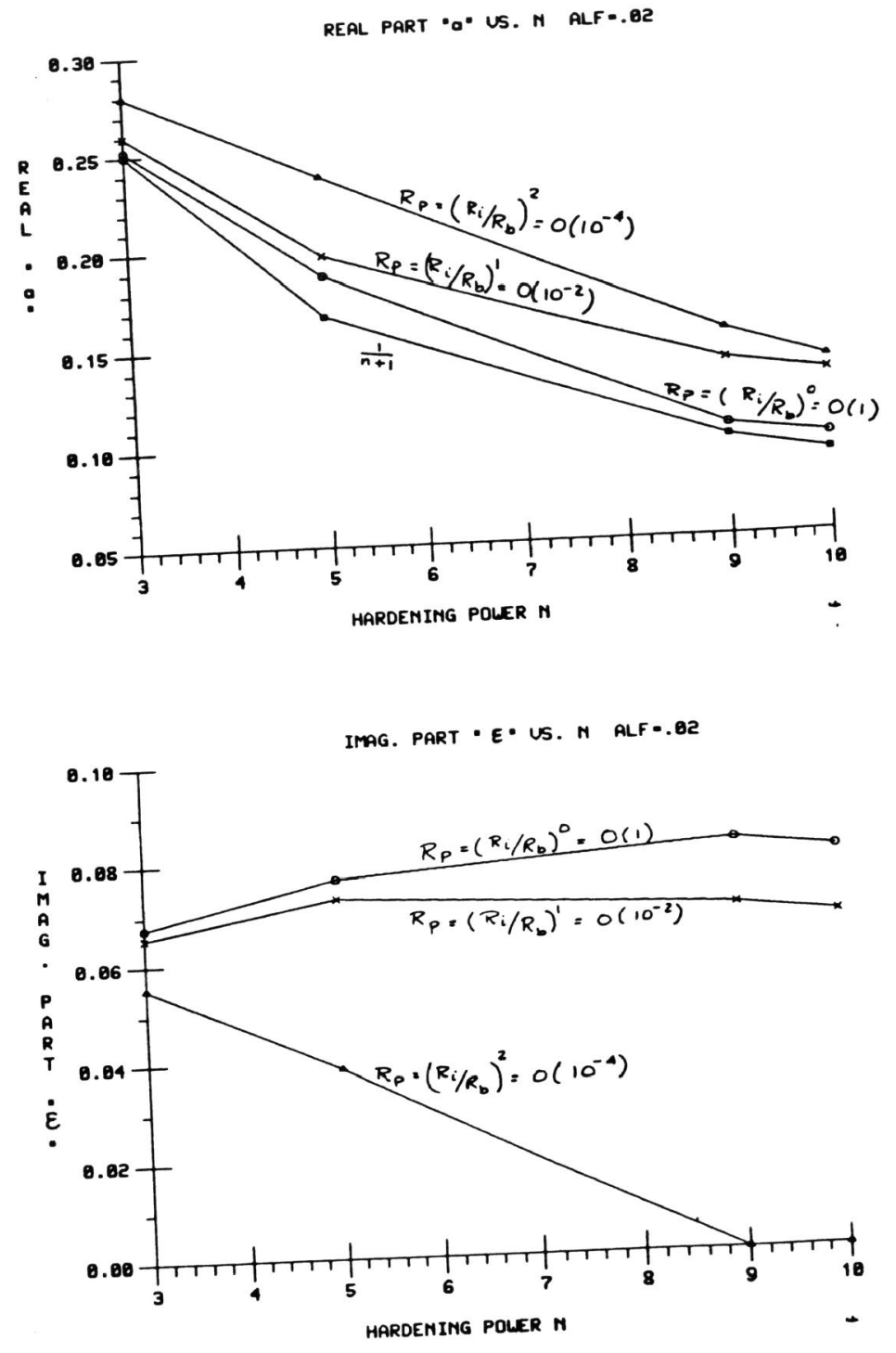

Fig. 2 Singularity Power for Process Zones $O(1), O\left(10^{-2}\right), O\left(10^{-4}\right)$ 

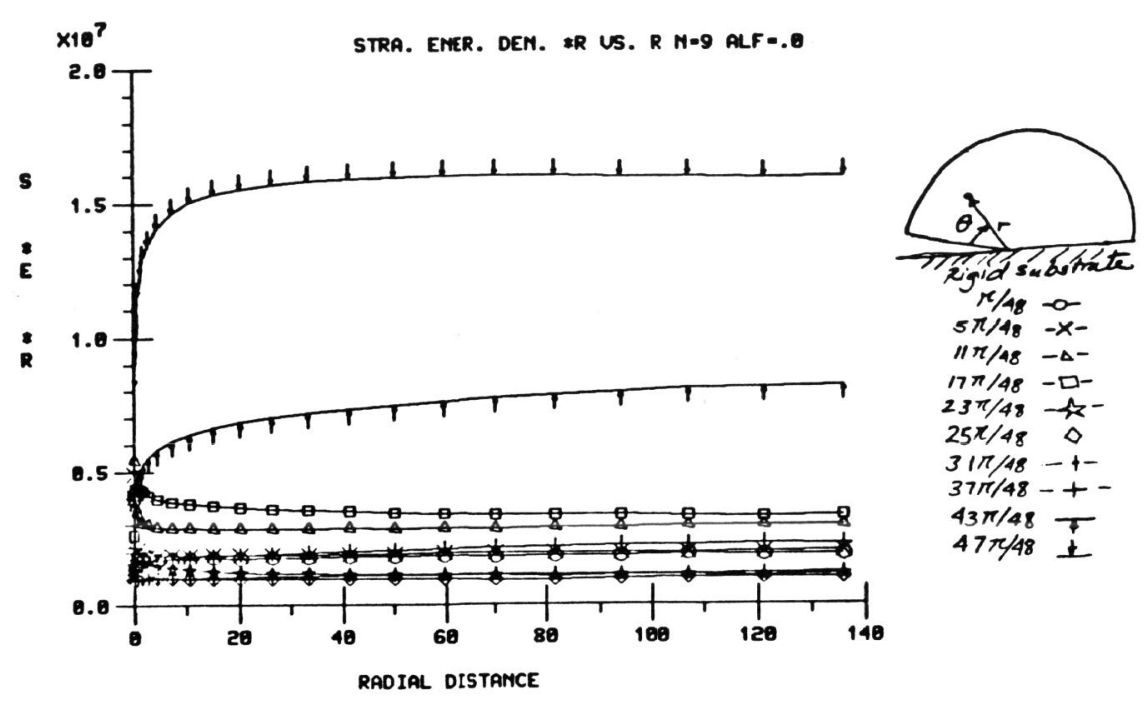

Fig. $3 \quad \sigma_{i j} \epsilon_{i j} \cdot r$ vs. $r$
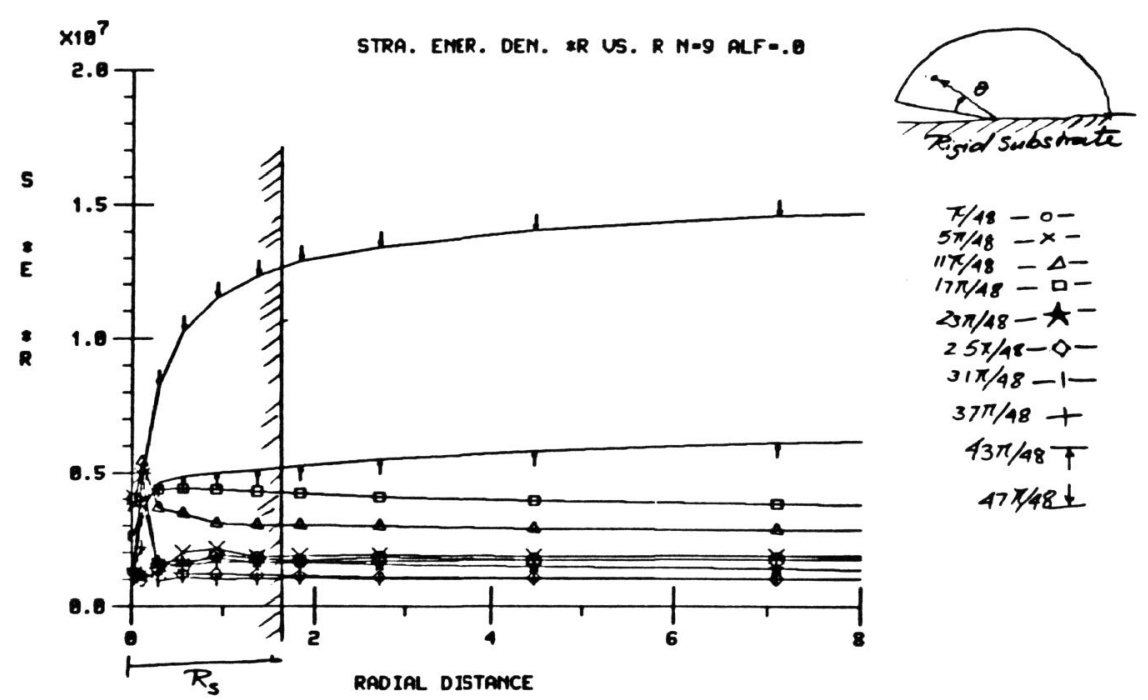

$\pi / 48-0-$

$11 \pi / 48=\Delta$

$17 \pi / 48=-$

$2 \pi / 48-A-$

$25 x / 48-0-$

$1 \pi / 48-1$

$47 \pi / 48+$

F1g. $4 \quad \sigma_{i j} \epsilon_{i j} \cdot r$ vs. $r$ 